\title{
OXIGENIOTERAPIA: O CONHECIMENTO DA EQUIPE DE ENFERMAGEM ${ }^{1}$
}

\section{NURSING KNOWLEDGE OF OXIGENOTHERAPY}

\section{Liandra Grando ${ }^{2}$ Cláudia Silveira Viera ${ }^{3}$}

\begin{abstract}
RESUMO: O estudo objetivou identificar o conhecimento da equipe de enfermagem sobre as seqüelas que a oxigenioterapia pode acarretar ao recém-nascido (RN) e como ocorre o cuidado de enfermagem nessa terapêutica. A pesquisa foi qualitativa sob o referencial de MINAYO (1996) aplicada a equipe de enfermagem de uma UTI neonatal. Denotando na categorização: o cuidado de enfermagem prestado ao $R N$ em oxigenioterapia e a percepção da equipe de enfermagem sobre as possíveis seqüelas ao RN em oxigenioterapia. Conclui-se que os cuidados são prestados sem uma padronização e o conhecimento acerca desse tratamento ainda é restrito.
\end{abstract}

PALAVRAS-CHAVE: Oxigenioterapia; Enfermagem; recém-nascido.

ABSTRACT: The purpose of this study was to identify the knowledge of the nurses' team about the sequels that the oxigentherapy may cause to the newborns and how to make the nurse's care. The research was qualitative according to MINAYO (1996) and applied to the nurse's team of an intensive care for newborns. The analysis was based in two categories: the nurse's care for the newborn in oxigentherapy and the nurse's team perception about some possible sequels to the newborn in oxigentherapy. It was possible to observe that the care was applied without any standardization and the knowledge about this problem is not enough.

KEY WORDS: Oxigentherapy; Nursing; Newborn.

\section{INTRODUÇÃO}

Todo ser vivo, para sua existência, realiza trocas com o meio ambiente, dentre elas destaca-se as trocas gasosas que ocorrem no ato de respirar.

O homem possui um sistema respiratório que Ihe possibilita a vida que começa desde seu desenvolvimento intra-uterino, especificamente ao redor de 26 a 28 dias após a fertilização. Com 16 semanas de gestação, o pulmão está morfologicamente parecido com o pulmão do adulto, por volta de 24 a 26 semanas de gestação, começa a produção do surfactante pulmonar, substância responsável pelo não colabamento dos alvéolos no processo respiratório. Assim, o pulmão fetal, mesmo imaturo, apresenta condições para trocas gasosas e síntese de surfactante, possibilitando a sobrevivência do RN com peso entre 500 e $1000 \mathrm{~g}$. A formação total dos alvéolos só se completa após dois meses do nascimento (ZUGAIB \& KANAS, 1986).

Os movimentos respiratórios encontram-se presentes na vida intra-uterina desde o final do primeiro trimestre gestacional, mas a respiração não ocorre com a função de oxigenação. Portanto, podemos dizer que é o preparo para a adaptação à vida extra-uterina que está ocorrendo. Pode-se afirmar que a respiração é fundamental para todos os seres vivos, com o objetivo de manter a integridade dos processos biológicos.

O trabalho de parto exerce importante função no estabelecimento da respiração. Neste momento iniciam-se os movimentos respiratórios propriamente ditos, desencadeados pela asfixia sofrida durante o trabalho de parto e pelos estímulos excitatórios que atuam sobre o centro respiratório, deflagrando os movimentos respiratórios extra-uterinos.

A necessidade mais crítica do recém-nascido (RN) é o estabelecimento da atividade respiratória adequada com uma troca eficiente de gases. $O$ estabelecimento da respiração é fundamental para a sobrevivência e a manutenção da integridade biofisiológica do RN. Então, quando há alguma falha no estabelecimento da respiração, pode-se necessitar de um tratamento especifico (NELSON, 1983).

$\mathrm{Na}$ falta de uma oxigenação adequada, ocorre a insuficiência respiratória que se manifesta por sinais de retrações costais, aumento do esforço respiratório, batimentos das aletas nasais, cianose, palidez, hipotensão e diminuição da perfusão periférica, apnéia com pausa respiratória seguida de bradicardia e ainda dispnéia. O RN deverá ser avaliado pelo profissional habilitado e, dependendo do caso a oxigenioterapia, poderá ser prescrita como tratamento.

\footnotetext{
${ }^{1}$ Trabalho de conclusão de curso de graduação em enfermagem.

${ }^{2}$ Enfermeira, graduada no curso de enfermagem da UNIOESTE.

${ }^{3}$ Enfermeira, Mestre em Enfermagem Fundamental, Docente da Área Materno Infantil da UNIOESTE - PR, e-mail: victorclaudia@uol.com.br; R. Bétula 124,Tropical, Cascavel-PR. 85802240.
} 
Assim, em casos de dificuldade nas trocas gasosas de ordem pulmonar, pode-se utilizar a terapia com $\mathrm{O} 2$ para tratamento da dificuldade respiratória. No entanto, deve-se considerar que complicações por alteração nas concentrações de $\mathrm{O} 2$ administrado ao RN podem causar seqüelas irreversíveis em algum período da infância, tal como alteração ocular podendo evoluir para cegueira (GARIJO, 2000).

Nas Unidades de Terapia Intensiva neonatal (UTI-Neo) utiliza-se muito a oxigenioterapia, portanto, os RN submetidos a esse tratamento são sujeitos passíveis de sofrerem complicações decorrentes do uso prolongado de $\mathrm{O} 2$.

A assistência de enfermagem é fundamental a esse RN para proporcionar segurança e eficácia no tratamento, em especial relacionado à toxicidade do O2, a qual pode levar a oxidação e morte celular. Conforme GUYTON \& HALL (1997, p. 962) coloca "descobriu-se que o uso de altas concentrações de oxigênio no tratamento de crianças prematuras, especialmente na prematuridade extrema, pode levar a cegueira. A razão disto é que oxigênio demais faz cessar o crescimento de novos vasos sangüíneos na retina". Muitas vezes essas complicações se instalam de modo sutil exigindo uma equipe experiente e bem preparada, para intervir precocemente, evitando complicações decorrentes desse processo.

Considerando esses aspectos complicadores da oxigenioterapia, percebe-se que a equipe de enfermagem deverá estar apta para prestar uma assistência adequada ao RN, ou seja, uma assistência que procure evitar ou diminuir possíveis seqüelas. Dessa forma, é preciso que a equipe de enfermagem tenha conhecimento das seqüelas decorrentes da oxigenioterapia e que saiba que são necessários cuidados específicos com esse tratamento, que poderão diminuir ou evitar complicações para o RN. Assim, se faz necessário conhecer como ocorre a assistência de enfermagem ao $\mathrm{RN}$ em oxigenioterapia hospitalizado em uma UTI-Neo.

Para REICHERT \& FOGLIANO (1995, p. 75) "a administração inadequada do oxigênio poderá ser iatrogênica para o RN, podendo resultar em lesões cerebrais, pulmonares, oculares e até mesmo a morte". Então, o cuidado com a oxigenioterapia é fundamental, pois a mesma consiste no estabelecimento das trocas gasosas pulmonares com o objetivo de manter o metabolismo celular adequado, assegurando a oxigenação do sangue arterial e garantindo a eliminação do dióxido de carbono (GARIJO, 2000).

Para RODRIGUES (1998) a toxicidade do O2 é atribuída às reações de radicais livres de $\mathrm{O} 2$ nos componentes celulares, mesmo que estes tenham papéis fundamentais no desenvolvimento normal dos processos biológicos e nas reações de oxidação e redução ao nível celular. Essas reações podem romper a membrana lipoprotéica, destruindo as funções enzimáticas celulares, mudando o DNA e levando a morte celular. A definição de radical livre é quando há um ou mais elétrons não pareados na órbita externa, tornando-se extremamente reativos, pois quando um radical livre reage com um radical não-livre, o produto é outro radical livre, produzindo reações em cadeia. Essa cadeia termina quando ocorre o encontro entre dois radicais livres que produzem uma ligação entre dois elétrons, ou com o encontro com uma substância antioxidante.

A retinopatia da prematuridade ainda não tem sua etiopatogenia conhecida completamente, sabe-se que é uma doença multifatorial, que afeta principalmente $\mathrm{O} R \mathrm{~N}$ de baixo peso e seu desenvolvimento se relaciona com o uso do $\mathrm{O} 2$ terapêutico e outros fatores de risco que ainda não foram totalmente esclarecidos como: RN prematuro, hiperóxia, uso de surfactantes, presença de ductos, alterações metabólicas, septicemia e curso clínico complicado (WAISMAN et al, 1999).

O desenvolvimento das lesões pulmonares esta diretamente relacionado à quantidade de $\mathrm{O} 2$ nos gases inspirados, quando a concentração de $\mathrm{O} 2$ administrada for inferior a 50\%, não ocorrem alterações pulmonares. Ressaltam ainda que, as lesões pulmonares, pelo uso de $\mathrm{O} 2$, encontram-se ligadas à doença de base. No caso da displasia broncopulmonar (DBP), que se caracteriza por alterações pulmonares mínimas até uma grande deterioração da função respiratória, a principal característica é a dependência do $\mathrm{O} 2$ pela insuficiência respiratória. Essa doença está relacionada ao uso de ventilação mecânica (VM) no tratamento da membrana hialina e também ao uso de altas concentrações de $\mathrm{O} 2$ sem VM, e suporte ventilatório artificial com baixa fração de oxigênio inspirado ( $\mathrm{FiO} 2)$. A FiO2 elevada e a VM com altas pressões inspiratórias e expiratórias, são fatores que agridem o pulmão do RN e contribuem para o desenvolvimento da doença (FREDDI \& TANNURI, 1993).

O RN submetido ao tratamento de oxigenioterapia está potencialmente sujeito as complicações descritas, exigindo um preparo da equipe profissional, que poderá estar identificando os sinais e sintomas para intervir de modo preventivo, ou mesmo para diminuir as complicações decorrentes de tratamento com $\mathrm{O} 2$.

Este embasamento anatomofisiológico, bem como o conhecimento do que há na literatura sobre as possíveis seqüelas da oxigenioterapia ao $\mathrm{RN}$, possibilita o planejamento da assistência de enfermagem.

TAMEZ (1999) coloca que, para o uso da oxigenioterapia deve-se estabelecer alguns critérios: a monitorização da concentração de $\mathrm{O} 2$ administrado e a saturação sangüínea de $\mathrm{O} 2$, e evitando, assim, a administração desnecessária ou de concentrações elevadas de $\mathrm{O} 2$, que poderão trazer efeitos tóxicos ao RN tanto a termo como no prematuro. Todo RN que estiver em tratamento de $\mathrm{O} 2$ deve estar conectado ao oxímetro de pulso, devendo-se avaliar periodicamente a gasometria arterial ou capilar, para avaliação dos níveis de $\mathrm{O} 2$ sangüíneo.

Há várias técnicas para administração da oxigenioterapia, a escolha é realizada pelo médico responsável, com base nos exames preliminares e na experiência da equipe, bem como da disponibilidade de aparelhos e recursos materiais no hospital. 
A assistência de enfermagem ao RN submetido a tratamento de $\mathrm{O} 2$ deve ser executada mediante um plano de cuidados baseado no processo de enfermagem, percebendo o paciente como um ser global, atendendo suas necessidades e de acordo com o método de administração de $\mathrm{O} 2$ utilizado. Com o processo de enfermagem, pode-se individualizar os cuidados com base científica, elevando a qualidade da assistência prestada e contribuindo para a recuperação mais rápida do paciente.

O RN submetido ao tratamento de oxigenioterapia exige cuidado especial e depende de uma equipe multidisciplinar, em especial da enfermagem, que acompanha 24 horas o RN em tratamento, exigindo assim profissionais especializados, preparados para diagnosticar e intervir com rapidez e eficiência nas intercorrências. Deste modo, pode-se prevenir as possíveis complicações deste tratamento e, assim, poder cumprir com o verdadeiro objetivo da oxigenioterapia que é promover oxigenação tecidual adequada.

Esta revisão da leitura acerca da temática levou a esse estudo, o qual teve como objetivos:

- Conhecer como ocorre a assistência de enfermagem ao $\mathrm{RN}$ submetido ao tratamento com $\mathrm{O} 2$;

- Identificar o conhecimento da equipe de enfermagem sobre as seqüelas que o tratamento pode acarretar ao RN.

\section{METODOLOGIA}

Este estudo foi desenvolvido por meio da pesquisa qualitativa, em que se buscou apreender da realidade vivida pela equipe de enfermagem que presta assistência ao RN em oxigenioterapia na UTI Neonatal, os dados que permearam esta pesquisa.

A pesquisa foi realizada mediante questões norteadoras com um instrumento previamente elaborado e enviado para aprovação do Comitê de Ética e Pesquisa com Seres Humanos da Instituição de Ensino de origem deste trabalho, recebendo parecer favorável para a execução. A população compôs-se por profissionais da equipe de enfermagem e a amostra por aqueles atuantes na UTI Neonatal, do Hospital Universitário do Oeste do Paraná, conforme aceitação prévia da instituição para participar.

A categorização dos dados se deu mediante o agrupamento de elementos, idéias ou expressões, ocorrendo então a partir das falas com características comuns. Optou-se no estudo por estar citando apenas trechos de falas mais significativas que representam a categoria proposta.

A análise das entrevistas foi baseada na análise de conteúdo, em que a análise do texto é baseada em critérios da teoria que serve de guia à leitura, descobrindo os núcleos de sentido da comunicação, cuja presença ou freqüência se correlacionem com o objetivo analítico (MINAYO, 1994). A partir do esclarecimento dos conceitos sobre categorização das entrevistas, parte-se para a etapa da análise das categorias e discussão das mesmas.

APRESENTAÇÃO E ANÁLISE DOS DADOS
A amostra deste estudo foi composta por funcionários que atuam na UTI Neonatal e berçário do HUOP. O número de entrevistados foi menor que 50\% do total de funcionários da unidade, pois houve certa resistência por parte deles em conceder as entrevistas, fato que certamente influenciou nos resultados deste trabalho.

Do total de funcionários atuantes na UTI-Neo, foram entrevistados oito funcionários, quatro do turno da manhã e 4 do turno da tarde, entrevistando-se então, $47 \%$ do total dos funcionários.

No agrupamento dos dados surgiram duas categorias denominadas: "o cuidado de enfermagem prestado ao RN em oxigenioterapia" e, a outra, "percepção da equipe de enfermagem sobre as possíveis seqüelas ao $\mathrm{RN}$ em oxigenioterapia”. Em cada categoria obteve-se duas subcategorias respectivamente, conforme se passa a discutir a seguir:

\section{O cuidado de enfermagem prestado ao RN em oxigenioterapia}

Nesta categoria todos os entrevistados citaram diferentes cuidados com o RN em oxigenioterapia ilustrado aqui pela fala da entrevistada rosa:

"temos várias formas de oferecer O2 ao RN...

ventilação mecânica... os cuidados de aspiração, cuidando para não cair à saturação da criança, observando pelo oxímetro... cuidando para não demorar demais, e assim não diminuindo a oxigenação".

Embasando-se nesses dados, obteve-se nesta categoria as seguintes subcategorias: descrevendo os cuidados de enfermagem com a oxigenioterapia e priorizando a conduta médica.

$\mathrm{Na}$ subcategoria descrevendo os cuidados de enfermagem com a oxigenioterapia, foram agrupados especificamente os cuidados pertinentes a equipe de enfermagem para o RN em oxigenioterapia. Nesse sentido, percebe-se que determinados cuidados rotineiros deixaram de ser citados, como um que é simples e importantíssimo, a lavagem das mãos antes e após a manipulação do RN.

Os cuidados com campânula foram citados por cinco entrevistados, que justificam este cuidado observando a instalação correta da campânula, bem como verificar se há algum escape de O2. A preocupação com a instalação da campânula é fundamental, pois se não estiver corretamente posicionada ocorrerá flutuações ou mesmo perda do nível de $\mathrm{O} 2$ que poderá evoluir para uma hipóxia do RN se não for diagnosticado em tempo.

Em caso de necessidade de retirar o RN da campânula, sempre providenciar uma fonte de $\mathrm{O} 2$ para ser colocada próximo de suas narinas, deste modo previne-se à oscilação da concentração do $\mathrm{O} 2$ e instalação de uma possível hipóxia (TAMEZ, 1999). Este cuidado fica explicito conforme azaléia relata:

"... quando se retira da incubadora e no início sempre fica em campânula, aí sempre temos 
que tirá-la um pouco para fazer a higiene, se a criança agüentar e ficar, a gente já dá banho no chuveirinho, daí comunicamos o médico que a criança já fica mais tempo sem O2...".

O cuidado de enfermagem como a observação constante da evolução do RN, exame físico, alterações das condições clínicas, deverão ser registrados no prontuário para o acesso de toda equipe atuante na UTI-Neo.

Ainda neste cuidado com campânula três entrevistados referem não colocar o $\mathrm{O} 2$ diretamente na pele, boca, olho e ouvido, como meio de prevenir seqüelas. GARIJO et al (2000) coloca que é importante verificar se o $\mathrm{O} 2$ não está fluindo diretamente sobre o rosto do $\mathrm{RN}$, pois dependendo do fluxo e do ajuste que é feito sobre o rosto do RN, pode-se atingir concentrações de até $100 \%$ de O2, ou mesmo impedir a fuga do dióxido de carbono, bem como se faz necessário realizar o controle ambiental e arterial do $\mathrm{O} 2$. Observando que o uso do sistema de campânula para a oxigenioterapia é incompatível com concentrações acima de $60 \%$ de O2, este método é indicado para o $\mathrm{RN}$ com estresse respiratório mínimo a moderado e com necessidade de concentrações menores de $60 \%$ e resultados de gasometria dentro dos padrões considerados normais. Nas bibliografias aqui consultadas não foram encontradas relações de seqüelas pelo contato direto do $\mathrm{O} 2 \mathrm{com}$ a pele, ouvido, olho e boca, ficando como sugestão o desenvolvimento de pesquisa sobre este assunto, considerando que $13 \%$ dos entrevistados fizeram esta correlação.

Nesse sentido, o que se tem conhecimento é que a retinopatia da prematuridade é um tipo de complicação que se desenvolve devido à fragilidade dos vasos sanguíneos retinianos, os quais ainda não alcançaram seu completo desenvolvimento. Assim, os efeitos tóxicos do $\mathrm{O} 2$ dependem do tempo de exposição, da pressão parcial do $\mathrm{O} 2$ no sangue arterial, a toxicidade atua sobre os vasos retinianos causando vasoconstrição comprometendo seu desenvolvimento normal, podendo causar desde pequenas alterações na vascularização da retina até o descolamento da mesma (TAMEZ, 1999).

Um dos entrevistados citou a preocupação com a falta de um oftalmologista neonatal no hospital, para fazer o acompanhamento do RN em oxigenioterapia que tem o risco aumentado de desenvolver a retinopatia da prematuridade.

Outro cuidado detectado é o uso em incubadora do $\mathrm{O} 2$ umidificado mas não aquecido devido à falta de aquecedor de $\mathrm{O} 2$ disponível na UTI-Neo deste hospital. Observa-se então, que o funcionário é conhecedor da necessidade de aquecer o 02 além de umidificá-lo, no entanto, não há o equipamento adequado para tal procedimento, com isso muitas vezes o não desenvolvimento do serviço fica atrelado à falta de recursos materiais que impossibilitam o bom desempenho dos trabalhos. A literatura de enfermagem preconiza o aquecimento da água ente $32^{\circ} \mathrm{C}$ a $36^{\circ} \mathrm{C}$ da água para proporcionar uma mistura gasosa de $\mathrm{O} 2$ e ar aquecido e umidificado para o RN (TAMEZ, 1999; REICHERT\& FOGLIANO, 1995).

Foi citado no cuidado com o umidificador, que é fundamental a identificação da água, mas não houve menção sobre a troca desta, a cada 24 horas, bem como a troca do circuito a cada 48 horas, sendo este conjunto de cuidados importantíssimo para prevenir infecções pela colonização de bactérias hidrofílicas (TAMEZ, 1999).

O método de administração da oxigenioterapia por cateter nasal consiste em oferecer $\mathrm{O} 2$ introduzindo-se o cateter em uma das narinas e através deste é oferecido $\mathrm{O} 2$ ao RN. Neste método identificou-se como cuidados, não manter sempre o cateter na mesma narina, evitando-se ou mesmo diminuindo a lesão da mucosa nasal, bem como se deve mantê-la limpa, permitindo o fluxo adequado dos gases. Além desses cuidados citados deve-se também aspirar as vias aéreas superiores para evitar obstrução do cateter; umidificar a narina em que está o cateter com duas gotas de soro fisiológico a cada duas horas; ajustar o fluxo dos gases conforme prescrição médica; realizar o registro dos cuidados e proceder aos demais cuidados ao RN e oxigenioterapia (OJEDA et al, 1992).

Quanto aos cuidados com o RN em ventilação mecânica foram citados: observação da quantidade do O2 prescrito; do nível da água para manter o 02 umidificado, evitando-se a irritação de mucosa; aspiração da cânula endotraqueal e cavidade oral conforme a necessidade; cuidados com a saturação do $\mathrm{O} 2$; comunicar o médico às alterações do RN e identificação da necessidade de fazer acompanhamento da gasometria arterial.

A aspiração da cânula endotraqueal tem por finalidade a remoção das secreções das vias respiratórias e prevenção da obstrução da cânula, deverá ser realizada conforme a necessidade com técnica asséptica de acordo com a rotina estabelecida na unidade. Este procedimento se faz necessário nos RN entubados, não devendo ser realizado sem a avaliação dos ruídos respiratórios que indiquem a presença de secreções pulmonares; das alterações nos níveis de oxigenação e presença de agitação do RN. Portanto, a aspiração de rotina deve ser evitada, pois interfere no repouso e sono do $\mathrm{RN}$ e aumenta o risco de infecções. O procedimento deverá ser realizado com dois profissionais treinados, com técnica asséptica, limitando o tempo de duração, e assim, evitando a agitação do RN e conseqüentemente aumento da pressão arterial e intracraniana, que favorecem as hemorragias intraventriculares nos RN, especialmente nos RN prematuros.

Essas observações são de extrema importância na ventilação, pois o nível da água deve ser rigorosamente monitorado, porque se o nível da água baixar poderá ocorrer um aquecimento dos gases acima da temperatura ideal, que é de $32^{\circ} \mathrm{C}$ a $36^{\circ} \mathrm{C}$. A umidificação e o aquecimento adequado da água previne o ressecamento da mucosa das vias aéreas, bem como auxilia na manutenção da temperatura corporal e fluidifica as secreções, facilitando a sua remoção (TAMEZ, 1999; LACERDA \& CASTELI, 1993). 
TAMEZ (1999) coloca ainda que para o uso da oxigenioterapia deve-se estabelecer alguns critérios, como avaliação da monitorização da concentração de O2 administrado e a saturação sangüínea de $\mathrm{O} 2$, evitando-se a administração desnecessária ou concentrações elevadas desse gás, que poderão trazer efeitos tóxicos ao RN. Um cuidado que deve ser dispensado a todo RN que estiver em tratamento de O2 é mantê-lo conectado ao oxímetro de pulso, avaliando periodicamente a gasometria arterial ou capilar, para verificação dos níveis de $\mathrm{O} 2$ sangüíneo.

Percebe-se mediante o agrupamento feito nessa pesquisa que não houve uma fala conjunta sobre os cuidados de enfermagem para o RN, cada um centrouse mais nos cuidados específicos para o método de oxigenioterapia empregado e não nos cuidados fundamentais que propiciam a prevenção das possíveis seqüelas dessa terapêutica.

Dessa forma, apresenta-se o cuidado geral ao RN em oxigenioterapia segundo OJEDA et al (1992) que consiste na lavagem das mãos antes e depois do contato com o RN; checar prescrição médica quanto ao meio de fornecimento de $\mathrm{O} 2$ e sua quantidade; verificar freqüentemente o fluxo de $\mathrm{O} 2 \mathrm{e}$ ar observando vazamentos ou oscilações; manter o nível da água indicado no umidificador de $\mathrm{O} 2$; lavar o frasco diariamente e trocar a água, identificando-a com data, hora e assinatura de quem trocou; controlar sinais vitais freqüentemente no mínimo de três em 3 horas ou conforme prescrição do enfermeiro; registrar os sinais vitais, bem como os parâmetros prescritos para a oxigenioterapia; manter decúbito elevado do RN com coxim sobre os ombros facilitando a oxigenação; mudança de decúbito a cada duas horas; aspiração das vias aéreas superiores sempre que necessário; evitar roupas apertadas ou cobertores pesados, pois interferem na expansão torácica; realizar higiene diária de acordo com as condições do RN e Conforme orientação do enfermeiro; chamar o laboratório imediatamente quando solicitada gasometria e esperar pelo menos 15 minutos após a instalação da oxigenioterapia para coleta de sangue para este exame; observar os cuidados específicos de acordo com o meio de administração de $\mathrm{O} 2$; estimular o contato dos pais; em caso de transferência do RN para outros setores o enfermeiro deverá acompanhar a transferência; fazer o registro de cada procedimento realizado e as observações na folha de controles; diminuir a concentração de $\mathrm{O} 2$ gradualmente na campânula, oxitenda, croupette, conforme prescrição médica e comunicar imediatamente ao enfermeiro qualquer alteração.

A equipe de enfermagem deverá colocar em prática todos os seus conhecimentos científicos, auxiliando na avaliação dos sinais e sintomas, interpretando seus significados e interagindo com as outras informações diagnósticas, para propiciar os cuidados adequados ao RN em oxigenioterapia e prevenir as possíveis seqüelas que esta pode ocasionar.

Nesse contexto surge outra subcategoria denominada priorizando a conduta médica, a qual surge das colocações sobre a execução da prescrição médica como o único cuidado para o $\mathrm{RN}$ em oxigenioterapia.

Essas colocações podem ser mais bem

ilustradas pelas seguintes citações:

"Só administramos a oxigenioterapia quando é prescrita" (azaléia);

"Ocorre a critério do pediatra, o que ele manda fazer a gente faz (...)" então o médico vem $e$ aumenta, colhe gasometria, faz exames e através disso muda o O2...através dos exames ele pode fazer alteração da concentração do $\mathrm{O} 2$ ou medicar para mudar a acidose. "(camélia).

Considerando-se que a equipe de enfermagem não tem autonomia para fazer uso da oxigenioterapia sem a prescrição médica, salvo em caso de emergências, essas colocações são pertinentes, porém a questão feita era sobre quais os cuidados de enfermagem que eram prestados ao RN em oxigenioterapia nessa unidade. Esses entrevistados detiveram-se apenas no cumprimento da prescrição médica e não mencionaram os cuidados que a enfermagem deve ter nessa situação. Assim, percebese a supervalorização do trabalho e conhecimento do profissional médico, demonstrando a fragilidade da equipe de enfermagem perante seu papel na assistência ao paciente.

A enfermagem acompanha $\mathrm{R}$ RN em oxigenioterapia, portanto, conhece e deverá conhecer os procedimentos, técnicas e conseqüências positivas ou negativas que poderão advir do tratamento de oxigenioterapia tanto para seguir a prescrição como para contesta-la ou até mesmo auxiliar na mudança de conduta.

Checar a prescrição médica é um dos cuidados gerais citados por OJEDA et al (1992), assim como o meio de oferecer $\mathrm{O} 2 \mathrm{e}$ a sua quantidade, para se obter um bom desempenho dos cuidados prestados ao paciente é necessário que a equipe de enfermagem e médica procurem estar sempre em sintonia.

Segundo TROSTER \& TOMA (1996) o enfermeiro atua na supervisão e coordenação da sua equipe, bem como com a equipe médica e, juntos, estarão prestando um atendimento eficiente $\mathrm{e}$ organizado ao paciente.

Para o desenvolvimento destas tarefas, o enfermeiro deverá planejar suas ações mediante utilização do processo de enfermagem. Para ABE et al (1982, p. 23) "o processo de enfermagem, além de individualizar o cuidado, melhorando a qualidade da assistência, evita que esta seja baseada apenas em rotinas e cumprimento de ordens médicas; informa, orienta e oferece oportunidade de participação dos membros da equipe no planejamento". Nos resultados desse estudo não houve menção da prescrição de enfermagem como um meio de seguir os cuidados para o RN em oxigenioterapia, somente a prescrição médica foi citada. A prescrição de enfermagem faz parte do processo de enfermagem e deverá ser desenvolvida diariamente para cada paciente, para assisti-lo na sua individualidade e integralidade. Dessa forma com a prescrição de enfermagem poderia se ter o planejamento dos cuidados a serem prestados para 
- RN em oxigenioterapia, evitando-se que cada funcionário haja conforme sua prática.

\section{A percepção da equipe de enfermagem sobre as possíveis complicações ao RN}

Nesta categoria foram agrupados os dados que os entrevistados citaram acerca das possíveis complicações ao RN decorrente da oxigenioterapia. A partir desta categoria surgiram as seguintes subcategorias: identificando as possíveis seqüelas da oxigenioterapia e não tendo certeza das seqüelas.

No que se refere a identificar possiveis seqüelas da oxigenioterapia, sete dos oito entrevistados citou diferentes possíveis complicações. Então, nesta subcategoria estão agrupados os dados referentes ao conhecimento dos funcionários acerca das possíveis complicações ao RN submetido a oxigenioterapia.

Margarida coloca que o O2 administrado diretamente no olho e ouvido do $\mathrm{RN}$, pode trazer complicações irreversíveis, e ainda que segundo os pediatras pode trazer dificuldades no aprendizado, causar cegueira e surdez.

Das bibliografias consultadas não se encontrou relação entre a dificuldade do aprendizado da criança ou surdez e o uso da oxigenioterapia, tem-se conhecimento sim de que a retinopatia da prematuridade é uma das complicações decorrente da exposição do RN a elevadas concentrações de $\mathrm{O} 2 \mathrm{e}$ por tempo prolongado. O O2 causa vasoconstrição nos vasos retinianos, não pelo fato de estar direcionado ao olho do RN, mas sim devido ao excesso do $\mathrm{O} 2$ no sangue arterial.

As elevadas taxas de concentração de $\mathrm{O} 2$ e o tempo de exposição estão diretamente ligadas à lesão da retina. Devido à fragilidade dos vasos capilares da retina e também porque não atingiram a sua maturidade, então o $\mathrm{O} 2$ em concentrações elevadas e em pressões atmosféricas aumentadas podem afetar a estabilidade do glóbulo vermelho e alterar o cérebro do $\mathrm{RN}$. Os efeitos tóxicos do $\mathrm{O} 2$ também se relacionam ao estágio de desenvolvimento dos vasos da retina, ao tempo de exposição ao $\mathrm{O} 2$, a pressão parcial do $\mathrm{O} 2$ no sangue arterial. Quando o tempo de exposição do RN ao $\mathrm{O} 2$ é por períodos curtos, os efeitos da oxigenioterapia são reversíveis. A vasoconstrição não é observada quando a retina encontra-se bem vascularizada, portanto, quanto menor for a idade gestacional maior será o risco de desenvolver a retinopatia da prematuridade devido à fragilidade dos vasos retinianos na porção temporal. Como prevenção da lesão da retina à pressão do $\mathrm{O} 2$ deve ser mantida abaixo do nível que estimula a vasoconstrição, no entanto, a concentração tóxica exata, ainda, não é conhecida. Para o prematuro pequeno e em bom estado, recomenda-se o uso de $40-60 \mathrm{mmHg}$ (KLAUS et al, 1982; TAMEZ, 1999; WAISMAN et al, 1999).

Dois dos entrevistados citaram a lesão ocular como uma das mais graves seqüelas que acometem o $\mathrm{RN}$, e que a sua causa é decorrente não do $\mathrm{O} 2$ direcionado ao olho do $\mathrm{RN}$, mas pela exposição a elevadas concentrações e por períodos prolongados. Esta afirmação dos entrevistados é confirmada na exposição feita acima sobre 0 processo de desenvolvimento da retinopatia da prematuridade, demonstrando assim o conhecimento da verdadeira causa dessa complicação.

O risco para o desenvolvimento do pneumotórax em RN que fazem uso da oxigenioterapia foi citado por gérbera:

“... que de $100 \%$, 90\% é o risco maior de um pneumotórax...".

Nas bibliografias consultadas não encontramos dados sobre a porcentagem de incidência do pneumotórax em RN submetidos a oxigenioterapia, para compará-los com a fala, no entanto, BRADY \& GREGORY (1982) observaram que os RN que fazem uso da oxigenioterapia em ventilação mecânica têm maior risco de desenvolvê-lo, devido às altas pressões do $\mathrm{O} 2$.

O pneumotórax se instala após a ruptura alveolar, e o ar fica preso no espaço interpleural, essa retenção de ar causa hiperdistensão pulmonar, ocasionando graus variáveis de colapso pulmonar. Os sinais clínicos do pneumotórax são cianose, taquipnéia, assimetria torácica, apnéia, bradicardia, gemência e batimento das aletas nasais. Os RN prematuros submetidos à ventilação mecânica têm maiores chances de desenvolver um pneumotórax (PRONCIANOY, 1997).

O acompanhamento da gasometria arterial foi colocado como uma necessidade para fazer uma avaliação da real necessidade da quantidade do $\mathrm{O} 2$, e se caso este controle não for realizado podem ocorrer complicações imediatas ou tardias pelo excesso ou mesmo pela falta do $\mathrm{O} 2$. Este acompanhamento se torna fundamental, pois os gases sanguíneos arteriais refletem o estado pulmonar, cardíaco e metabólico do RN. Todos os RN submetidos a oxigenioterapia deverão ser conectados ao oxímetro de pulso e periodicamente avaliados quanto à gasometria arterial ou capilar para avaliação dos níveis de $\mathrm{O} 2$ no sangue. Estes cuidados devem ser tomados independente do método de tratamento com O2 ao RN (KLAUS et al, 1982; TAMEZ, 1999).

Do total de entrevistados dois associam complicações decorrentes do uso do $\mathrm{O} 2 \mathrm{em}$ concentrações elevadas e tempo de exposição prolongado, ou mesmo decorrente da sua falta.

Enumera-se neste estudo as complicações mais freqüentes que acometem o RN submetido a oxigenioterapia, devido aos efeitos tóxicos dos radicais livres de 02: displasia broncopulmonar, retinopatia da prematuridade, enterite necrosante e hemorragia intracraniana. Quando há falta do O2 ocorre uma hipoventilação, este estado pode não causar alterações na circulação pulmonar, no entanto, a ventilação alveolar é inadequada para um determinado nível de metabolismo devido à diminuição do volume por minuto. A hipoventilação pode levar a uma hipoxemia quando os valores da saturação da hemoglobina forem inferiores a $90 \%$. A hipoventilação pode ser classificada em central ou periférica, sendo que a avaliação da hipoxemia pode ser realizada através dasaturação da hemoglogina (RODRIGUES, 1998; PIVA et al, 1998). 
No caso do RN submetido a oxigenioterapia em ventilação mecânica (VM) as complicações mais freqüentes são displasia broncopulmonar, infecção secundária pulmonar, atalectasia pós-extubação, obstrução laríngea, pneumotórax, hemorragia pulmonar, alterações no equilíbrio ácido básico (TROSTER \& TOMA, 1996; PRONCIANOY, 1997).

Dentro da subcategoria não tendo certeza das seqüelas foram agrupados os dados referentes à incerteza da ocorrência de seqüelas, bem como o desconhecimento das mesmas.

Percebeu-se que há a preocupação da equipe com as seqüelas, no entanto, não há uma certeza do modo que elas se instalam e seus sinais de manifestação. Cincos dos entrevistados colocaram que o uso do $\mathrm{O} 2$ poderia estar trazendo complicações pulmonares, infecções secundárias, lesões cerebrais e diafragmáticas. Apenas um dos entrevistados relatou não saber se há ou não a ocorrência de problemas referentes ao uso do $\mathrm{O} 2$.

Rosa comenta que o RN pode sofrer uma:

“... atalectasia pós-extubação, edema devido ao tempo de entubação, podendo trazer infecção geralmente secundária...".

Estas são complicações citadas pela literatura como passíveis de acometer o RN em oxigenioterapia, a atalectasia ocorre devido ao acúmulo de secreção que ocorre com freqüência após a extubação; o edema de laringe pode ocorrer devido ao tempo prolongado de entubação e ainda pode causar obstrução de laringe; a infecção secundária pulmonar é comum no RN que permanece nesta situação por tempo prolongado (PRONCIANOY, 1997).

As complicações diafragmáticas, na maioria das vezes são decorrentes do próprio estado clínico do $\mathrm{RN}$ que o deixa mais susceptível a desenvolver uma insuficiência respiratória, principalmente quando há uma diminuição da função da musculatura diafragmática e intercostal menos madura, ocorrendo geralmente em RN prematuros ou desnutridos. Este fato contribui para a exaustão e esgotamento precoces da massa muscular diafragmática, quando é solicitado que trabalhe acima de suas condições. A ocorrência da apnéia é comum neste quadro e a patologia pulmonar geralmente associada é a doença da membrana hialina (PIVA et al, 1998).

O desmame brusco do respirador foi relacionado com possíveis complicações, este deve ser realizado de acordo com o acompanhamento dos gases sanguíneos, bem como outros exames complementares e o exame clínico. Dentre os cuidados com o desmame é preciso fazer uma minuciosa avaliação das condições cardiorespiratórias e metabólicas estáveis, que consiste em diminuir gradativamente a assistência da VM e na concentração do $\mathrm{O} 2$ e com a monitoração dos gases sangüíneos e aspectos clínicos até a ventilação espontânea. Deve-se ter o cuidado de não agitar o RN imediatamente antes da extubação; manter jejum de seis horas antes, prevenindo aspirações do conteúdo gástrico; o material para uma possível reentubação deverá sempre estar preparado. Depois de realizada a extubação o RN deverá ficar em ar ambiente com O2 umidificado e aquecido, de 10 a $20 \%$ acima do que estava exposto antes da extubação, podendo usar a oxitenda ou máscara.

O decúbito elevado é preciso ser mantido e fazer rigoroso controle de alterações clínicas ou gasométricas. A aspiração das vias aéreas pode ser realizada conforme a necessidade (LACERDA \& CASTELLI, 1993).

\section{CONSIDERAÇÕES FINAIS}

Os objetivos propostos no estudo foram alcançados, percebendo-se que a equipe de enfermagem tem $\mathrm{O}$ conhecimento dos meios de oferecer $\mathrm{O} 2$ ao $\mathrm{RN}$, no entanto, no que se refere a cuidados específicos dessa terapêutica, não houve consenso entre as falas. Foram citados alguns cuidados de acordo com o método utilizado para oferecer $\mathrm{O} 2$ e não foram citados os cuidados gerais com essa terapêutica, que deveriam estar presente nas falas com maior freqüência.

Identificou-se também que os cuidados de enfermagem são realizados de acordo com o conhecimento que cada um acredita ser correto, devido à falta de homogeneidade nas falas. Algumas seqüelas que podem acometer $\circ$ RN em oxigenioterapia foram citadas, como a retinopatia da prematuridade, mas com distorções em relação ao modo de como se instala.

A preocupação em seguir a prescrição médica é grande, devendo-se considerar sim, que checar a prescrição médica, quanto ao meio de oferecer $\mathrm{O} 2 \mathrm{e}$ sua quantidade faz parte dos cuidados gerais de enfermagem, mas também se deve lembrar que a enfermagem assiste $\mathrm{o}$ paciente na sua totalidade, e para tanto, poderá valer-se do processo de enfermagem, que atua como um instrumento facilitador das ações planejadas, para que através deste seja possível informar, orientar e oferecer oportunidade para que toda equipe de enfermagem participe do planejamento da assistência. Menciona-se o uso do processo, como um meio de planejar e implementar a assistência de enfermagem ao RN em oxigenioterapia, pois neste estudo não foram citadas nenhuma das etapas deste.

Observou-se que a falta de equipamentos adequados também interfere na assistência, como por exemplo, no caso o oferecimento de 02 é umidificado e, mas não é aquecido. O entrevistado citou o aquecimento do $\mathrm{O} 2$ como uma necessidade, no entanto, o hospital não oferece 0 equipamento adequado para tal procedimento. A estrutura física $e$ de recursos humanos esta diretamente ligada ao sucesso e ao bom desempenho das ações do cuidado de enfermagem.

\section{REFERENCIAS BIBLIOGRAFICAS}

ABE, E. S. H.; HIRAYAMA, A. F.; AMADEI, M. C. V. Assistência de enfermagem na insuficiência respiratória. 
In: CURSINO, M. R. et al. Assistência de enfermagem em pediatria. São Paulo, Sarvier, 1992.

BRADY, J. P.; GREGORY, G. A. Ventilação assistida. In: KLAUS, M.; FANAROFF, A. Alto risco em neonatalogia. $2^{\mathrm{a}}$ ed., Rio de Janeiro, Interamericana, 1982.

FREDDI, N. A.; TANNURI, U. Toxicidade do oxigênio e displasia broncopulmonar. In: GARIJO, C. Pediatria. Rio de Janeiro, Mc Graw Hill, 2000.

GUYTON, A. C.; HALL, J. E. Tratado de fisiologia médica. 9 ed., Rio de Janeiro, Guanabara Koogan, 1997.

KLAUS, M.; FANAROFF, A. Alto risco em neonatalogia. $2^{\mathrm{a}}$ ed., Rio de Janeiro, Interamericana, 1982.

LACERDA, D. P. D.; CASTELLI, M. Assistência de Enfermagem à criança sob ventilação pulmonar mecânica. In: CARVALHO, W. B. et al. Ventilação pulmonar mecânica e pediátrica. São Paulo/Rio de Janeiro/ Belo Horizonte, Atheneu, 1993.

MINAYO, M. C. (Org.). Pesquisa social: teoria, método e criatividade. Petrópolis, RJ, Vozes, 1996.

NELSON, W. E. et al. Tratado de pediatria. 11 ed., Rio de Janeiro, Interamericana, 1983.

OJEDA, B. S. et al. Oxigenioterapia. In: BIEHL, J. et al. Manual de enfermagem pediátrica. Rio de Janeiro, Medsi, 1992.

PIVA, J. P.; GARCIA, P. C. R.; SANTANA, J. C. B.; BARRETO, S. S. M. Insuficiência respiratória na criança. Disponível em: <http://www.sbp.com.br/jornal/9811.12s/artll.html\#rodape> Acesso em: 07 abr. 2001.

PRONCIANOY, R. S. Problemas respiratórios. In: MIURA, E. et al. Neonatologia: princípios e prática. $2^{\mathrm{a}}$ ed., Porto Alegre, Artes Médicas, 1997.

REICHERT, M. C. F.; FOGLIANO, R. R. F. Assistência de enfermagem ao recém-nascido em oxigenioterapia. In: NAGANUMA, $M$. et al. Procedimentos técnicos de enfermagem em UTI neonatal. São Paulo/Rio de Janeiro/Belo Horizonte, Atheneu, 1995.

RODRIGUES, F. P. M. Importância dos radicais livres de oxigênio no período neonatal. Jornal de Pediatria, Rio de Janeiro, 74, p. 97-98 mar./abr. 1998.

TAMEZ, R. N; SILVA, M. J. P. Enfermagem na UTI neonatal: assistência ao recém-nascido de alto risco. $1^{\mathrm{a}}$ ed., Rio de Janeiro, Guanabara Koogan, 1999.

TROSTER, E. J.; TOMA, E. Insuficiência respiratória. In: LEONE, C. R.; TRONCHIN, D. M. R. Assistência integrada ao recém-nascido. São Paulo, Atheneu, 1996. ZUGAIB, M.; KANAS, M. Fisiologia fetal aplicada. $1^{\text {a }}$ ed., São Paulo, Livraria Roca LTDA, 1986.

WAISMAN, I.; LARRIESTRA, A.; ZABALO, S.; MONGIAT, $M$. Factores de riesgo en la retinopatia del prematuro. Disponivel em: http://www.sbp.com.br/jornal/9911.12/artseconsull.html Capturado em: 07 abr. 2001.
Texto original recebido em: $15 / 08 / 2002$ Aprovação final: 10/12/2002 